\title{
HARTOGS EXTENSION THEOREMS ON STEIN SPACES
}

\author{
NILS ØVRELID AND SOPHIA VASSILIADOU
}

\begin{abstract}
We discuss various known generalizations of the classical Hartogs extension theorem on Stein spaces with arbitrary singularities and present an analytic proof based on $\bar{\partial}$-methods.
\end{abstract}

\section{INTRODUCTION}

The classical Hartogs extension theorem is usually stated as follows:

Theorem 1.1. (Hartogs) Let $\Omega$ be a domain in $\mathbb{C}^{n}(n>1)$ and let $K$ be a compact subset of $\Omega$ such that $\Omega \backslash K$ is connected. Then any holomorphic function $f$ on $\Omega \backslash K$ has a unique holomorphic extension on $\Omega$.

The first analytic proofs of this theorem for $\Omega \subset \subset \mathbb{C}^{n}$ used the theory of integral kernels. The shortest proof so far of Theorem 1.1 is due to Ehrenpreis and relies on solvability with compact support of the CauchyRiemann equations for $\bar{\partial}$-closed smooth compactly supported $(0,1)$-forms defined in $\mathbb{C}^{n}$. Vanishing results of this type are instrumental in obtaining Bochner type extension theorems as well. Complex manifolds $X$ that possess this property i.e. $H_{c}^{1}(X, \mathcal{O})=0$ are often said to have the Bochner-Hartogs property. Examples of such manifolds are $(n-1)$-complete complex manifolds of dimension $n>1$ (this was shown by Andreotti-Grauert in [2] and Andreotti and Vesentini in 4]), and (n-1)-strictly-hyperconvex Kähler manifolds (proven by Grauert and Riemenschneider in 10). Various possible Hartogs extension theorems have appeared in the literature in which one can replace $\mathbb{C}^{n}$ by an $n$-dimensional Stein manifold $X$ or, replace the holomorphic sections by sections of a coherent sheaf on $X$. Similarly, various Bochner extension theorems have appeared in which one can replace $\mathbb{C}^{n}$ by a Stein or $(n-1)$-complete $n$-dimensional manifold (see section 3.4 in [3]). A weak form of Hartogs extension theorems for singular spaces first appeared in the seminal 1962 paper of Andreotti and Grauert (Théorème 15 in [2]). In 1963, Rossi proved the following version of a Hartogs extension theorem for normal Stein spaces using Bishop's special analytic polyhedra:

Theorem 1.2. (Theorem 6.6 in 23]) Let $X$ be a connected, normal Stein space of dimension $n \geq 2$. Let $K$ be a compact subset of $X$ and let $h$ be a holomorphic function on $X \backslash K$. There is a unique $H$ holomorphic on $X$ such that $H=h$ on the unbounded component of $X \backslash K$.

Later on Laufer, Harvey, Bănică and Stănăşilă generalized Rossi's result. Harvey showed in [16 the following

Theorem 1.3. (Theorem 2.13 in [16) Let $X$ be a subvariety in $\mathbb{C}^{n}$. Suppose $K$ is a compact subset of $X$ such that $X \backslash K$ has no branches which are contained in a compact set. If the homological codimension $\operatorname{codh}_{x}\left(\widetilde{\mathcal{O}}_{X}\right) \geq 2$ for all $x \in X$, then the restriction map

$$
\Gamma\left(U, \mathcal{O}_{X}\right) \rightarrow \Gamma\left(U \backslash K, \mathcal{O}_{X}\right)
$$

is bijective for all neighborhoods $U$ of $K$ in $X$.

Conversely, if $\Gamma\left(X \backslash K, \mathcal{O}_{X}\right) \cong \Gamma\left(X, \mathcal{O}_{X}\right)$ for a compact holomorphically convex subset of $\mathbb{C}^{n}$ with $K \subset X$, then $\operatorname{codh}_{x}\left(\widetilde{\mathcal{O}}_{X}\right) \geq 2$ for all $x \in K$. Here $\widetilde{\mathcal{O}}_{X}:=i_{*}\left(\mathcal{O}_{X}\right)$ is, the trivial extension of $\mathcal{O}_{X}$ by zero to $\mathbb{C}^{n}$, and $i: X \hookrightarrow \mathbb{C}^{n}$ is the closed immersion.

Key words and phrases. Cauchy-Riemann equation, Singularity, Cohomology groups. 2000 Mathematics Subject Classification: 32B10, 32J25, 32W05, $14 \mathrm{C} 30$.

The research of the second author is partially supported by NSF grant DMS-0712795. 
On another note in Bănică and Stănăşilă's book [5] the following generalization of Theorem 1.3 is given.

Theorem 1.4. (Chapter I, Corollary 4.4 in [5]) Let $(X, \mathcal{O})$ be a reduced Stein space and $K$ be a compact set such that $X \backslash K$ has no relatively compact irreducible components (branches) in $X$. If the homological codimension $\operatorname{codh}\left(\mathcal{O}_{X}\right) \geq 21$, then the restriction map

$$
\Gamma(X, \mathcal{O}) \rightarrow \Gamma(X \backslash K, \mathcal{O})
$$

is bijective.

Conversely, if $X$ is Stein and $K$ a holomorphically convex compact such that the map $\Gamma(X, \mathcal{O}) \rightarrow \Gamma(X \backslash$ $K, \mathcal{O})$ is bijective, then $\operatorname{codh}\left(\mathcal{O}_{x}\right) \geq 2$ for all $x \in K$.

In 2007, Merker and Porten established a version of a Hartogs extension theorem for $(n-1)$-complete (possibly singular) complex spaces using the method of pushing disks and Morse theory. More precisely they proved

Theorem 1.5. (Theorem 2.2 in [21]) Let $X$ be a connected $(n-1)$-complete normal space of dimension $n \geq 2$. Then, for every domain $\Omega \subset X$ and every compact set $K \subset \Omega$ with $\Omega \backslash K$ connected, holomorphic functions on $\Omega \backslash K$ extend holomorphically and uniquely to $\Omega$.

Ruppenthal proved in 24] Theorem 1.5 for 1-complete (Stein) spaces with isolated singularities, using the classical $\bar{\partial}$-method of Ehrenpreis, ideas from an earlier paper of ours [7] combined with Serre duality.

In the case of Stein spaces with singularities it is natural to examine the relationship of the above versions of the Hartogs extension theorem and also try to generalize Ehrenpreis approach for Stein spaces with arbitrary singularities. We shall show that Theorem 1.4 and Theorem 1.5 for Stein spaces are in fact equivalent; we will further show that Theorem 1.5 for Stein spaces implies the version of Theorem 1.4 under the weaker assumption that $\operatorname{codh}\left(\mathcal{O}_{x}\right) \geq 2$ for all $x \in K$. We shall also present in this paper an analytic approach to obtaining Hartogs extension theorems based on our earlier work. It may be worthwhile to state the following corollary which will tell us that only appropriate neighborhoods of $K$ need to be considered for extension theorems to hold:

Corollary 1.6. Let $(X, \mathcal{O})$ be a reduced complex analytic space $K$ be a compact subset of $X$ such that $\operatorname{codh}\left(\mathcal{O}_{X, x}\right) \geq 2$ for all $x \in K$. Let $D$ be an open neighborhood of $K$. If $K$ has a Stein neighborhood $U$ in $X$ such that $U \backslash K$ has no irreducible components that are relatively compact in $U$ then the restriction map

is bijective.

$$
\Gamma(D, \mathcal{O}) \rightarrow \Gamma(D \backslash K, \mathcal{O})
$$

The organization of the paper is as follows. In section 2, we will give an overview of the ideas used to prove Theorems 1.3, 1.4. In section 3 we will prove the equivalence of the various Hartogs extension theorems, the implication that Theorem 1.5 implies Corollary 4.4 as stated in [5] and show that the conditions imposed in Theorem 1.4 are necessary. In section 4 we will give a different proof of the Hartogs extension for Stein spaces $X$ that satisfy $\operatorname{codh}\left(\mathcal{O}_{X}\right) \geq 2$ based on Andreotti-Grauert's work. Section 5 will streamline the $\bar{\partial}$-approach.

Remark: This paper was completed in September of 2008. Around the same time Andersson and Samuelsson obtained in [1] (Theorem 1.4) a $\bar{\partial}$-proof of a Hartogs extension theorem on Stein spaces with arbitrary singularities, using residue theory calculus. In November of 2008, Coltoiu [6] and Ruppenthal [25] independently obtained a $\bar{\partial}$-theoretic proof of a Hartogs extension theorem on cohomological $(n-1)$-complete (resp. $(n-1)$-complete) spaces. The key ingredient in the proof is a vanishing of the higher direct images of the sheaf of canonical forms of an appropriate desingularization $\tilde{X}$ of the $(n-1)$-complete complex

\footnotetext{
${ }^{1}$ Corollary 4.4 on page 42 in [5] is stated under a weaker assumption on the homological codimension of the structure sheaf; namely that $\operatorname{codh}\left(\mathcal{O}_{x}\right) \geq 2$ for all $x \in K$. However a stronger assumption is needed for the proof of Corollary 4.4 in 5 to go through. One needs at least that $\operatorname{codh}\left(\mathcal{O}_{x}\right) \geq 2$ for all $x \in U$ where $U$ is a Stein neighborhood of $K$.
} 
space $X$. This subtle vanishing result which was obtained by Takegoshi [28, easily yields the vanishing of $H_{c}^{1}(\tilde{X}, \mathcal{O})$ which is needed for the Ehrenpreis method to carry over. Our analytic approach is based on more general weighted $L^{2}$-solvability results for $\bar{\partial}$-closed, compactly supported forms (Theorem 5.3) which are of independent interest. In a forthcoming work [22] we obtain semiglobal results for $\bar{\partial}$ on $q$-complete spaces and as a corollary we obtain another proof of a Hartogs extension theorem on $(n-1)$-complete spaces.

\section{Preliminaries}

2.1. Preliminaries on cohomology with support. Let us recall the obstructions to extending holomorphic sections from $X \backslash K$ to $X$. For a sheaf of abelian groups $\mathcal{S}$ on $X$, let $\Gamma_{K}(X, \mathcal{S})$ denote the sections on $X$ with support in $K$. Consider a flabby resolution of $\mathcal{O} ; \quad 0 \rightarrow \mathcal{O} \rightarrow \mathcal{C}^{0} \stackrel{d_{0}}{\rightarrow} \mathcal{C}^{1} \stackrel{d_{f}}{\rightarrow} \ldots$. The cohomology groups with support in $K$ are defined by $H_{K}^{*}(X, \mathcal{O}):=H^{*}\left(\Gamma_{K}(X, \mathcal{C} \bullet)\right.$, i.e. they are the cohomology groups of the complex $\left(\Gamma_{K}\left(X, \mathcal{C}^{k}\right), d_{k}\right)$. Since each $\mathcal{C}^{\bullet}$ is flabby, we have a short exact sequence $0 \rightarrow \Gamma_{K}\left(X, \mathcal{C}^{\bullet}\right) \rightarrow \Gamma\left(X, \mathcal{C}^{\bullet}\right) \rightarrow \Gamma\left(X \backslash K, \mathcal{C}^{\bullet}\right) \rightarrow 0$. This induces a long exact sequence on cohomology

$$
0 \rightarrow H_{K}^{0}(X, \mathcal{O}) \rightarrow H^{0}(X, \mathcal{O}) \rightarrow H^{0}(X \backslash K, \mathcal{O}) \rightarrow H_{K}^{1}(X, \mathcal{O}) \rightarrow H^{1}(X, \mathcal{O}) \rightarrow \ldots
$$

Since $X$ is a Stein subvariety $H^{i}(X, \mathcal{O})=\{0\}$ for all $i \geq 1$. Hence the above sequence becomes

$$
0 \rightarrow H_{K}^{0}(X, \mathcal{O}) \rightarrow H^{0}(X, \mathcal{O}) \rightarrow H^{0}(X \backslash K, \mathcal{O}) \rightarrow H_{K}^{1}(X, \mathcal{O}) \rightarrow 0
$$

It is clear from the above that the obstructions to extending holomorphic sections from $X \backslash K$ to $X$ come from nontrivial $H_{K}^{i}(X, \mathcal{O})$ for $i=0,1$.

It is a standard fact from sheaf cohomology theory that $H_{K}^{i}(X, \mathcal{O}) \cong H_{K}^{i}(U, \mathcal{O})$ where $U$ is an open neighborhood of $K$ in $X$. The fact that $H_{K}^{i}(U, \mathcal{O})$ is independent of the neighborhood $U$ of $K$ is referred to as excision.

Later on we shall need a more general version of (11). Let $L$ be a closed neighborhood of $K$ in $X$. Then we have the following exact sequence:

$$
0 \rightarrow H_{K}^{0}(X, \mathcal{O}) \rightarrow H_{L}^{0}(X, \mathcal{O}) \rightarrow H_{L \backslash K}^{0}(X \backslash K, \mathcal{O}) \rightarrow H_{K}^{1}(X, \mathcal{O}) \rightarrow H_{L}^{1}(X, \mathcal{O}) \rightarrow . .
$$

2.2. Preliminaries on homological codimension. Let us recall the definition and some properties of the homological codimension or profundity of a coherent sheaf. First of all,

- 1 On a noetherian ring $R$ with an $R$-module $M$ of finite type and $\alpha$ an ideal, a sequence of elements $f_{1}, \cdots, f_{q} \in \alpha$ is called a regular $M$-sequence if $f_{i}$ is not a zero divisor of $M / \sum_{j=1}^{i-1} f_{j} M$ (for $i=1$, it means that $f_{1}$ is not a zero divisor of $M$ ). The maximum length of a regular $M$-sequences is denoted by $\operatorname{prof}_{\alpha} M$. If $R$ is a local ring with maximal ideal $m$ then $\operatorname{prof}_{m} M$ is denoted by $\operatorname{codh}_{R} M$ and is called the homological codimension of $M$. An equivalent way to describe the homological codimension of a module $M$ of a local noetherian ring is the following: $\operatorname{codh}_{R} M=\inf \left\{i ; \operatorname{Ext}^{i}(k, M) \neq 0\right\}$ where $k=R / m$.

For a general complex space $(X, \mathcal{O})$ and a coherent analytic sheaf $\mathcal{F}$ on $X$, we define $\operatorname{codh} \mathcal{F}=\inf _{x \in X} \operatorname{codh}_{x} \mathcal{F}$ where $\operatorname{codh}_{x} \mathcal{F}=\operatorname{codh}_{\mathcal{O}_{x}} \mathcal{F}_{x}$, if $\mathcal{F}_{x} \neq 0$ and $\infty$ otherwise.

$\bullet_{2}$ If $i: X \rightarrow Y$ is a closed immersion of complex spaces and $\mathcal{F}$ is a coherent sheaf on $X$ then $i_{*}(\mathcal{F})$, the trivial extension of $\mathcal{F}$ by zero outside $X$, is a coherent sheaf in $Y$ and we have $\operatorname{codh}\left(\mathcal{F}_{x}\right)=\operatorname{codh}\left(i_{*}(\mathcal{F})_{i(x)}\right)$ for all $x \in X$.

Hartogs extension theorems for some singular Stein spaces follow as a special case of more general vanishing theorems of cohomology with supports. In what follows we outline the basic ideas behind these vanishing theorems. 
2.3. Vanishing on cohomology with supports and homological codimension bounds. The aim in this section is to recall the relationship between homological codimension bounds and vanishing of cohomology with supports.

Theorem 2.1. (Corollary 3.1, page 37 in [5]) If $X$ is a Stein space, $\mathcal{F}$ is a coherent sheaf on $X, K$ is a holomorphically convex compact and $r \geq 0$ an integer. Then $\operatorname{codh}\left(\mathcal{F}_{x}\right) \geq r+1$ for all $x \in K$ if and only if $H_{K}^{i}(X, \mathcal{F})=\{0\}$ for all $i \leq r$, where $H_{K}^{i}$ are the $i$-th cohomology groups with support on $K$.

Since $K$ is a holomorphically convex compact in a Stein space, it has a neighborhood basis of Oka-Weil domains i.e. $K$ has a Stein neighborhood that can be thought of as a holomorphic subvariety of a polydisk in some numerical affine space. So without loss of generality we can assume that $i: X \hookrightarrow \mathbb{C}^{n}$ is a closed immersion. Let $\mathcal{G}:=i_{*}(\mathcal{F})$, the trivial extension by zero outside $X$. The sheaf $\mathcal{G}$ is coherent. If $K$ is holomorphically convex subset of $X$ then $L:=i(K)$ is holomorphically convex compact in $\mathbb{C}^{n}$ and $H_{K}^{i}(X, \mathcal{F}) \cong H_{L}^{i}\left(\mathbb{C}^{n}, \mathcal{G}\right)$. Hence the proof of Theorem 2.1 reduces to the case of Stein manifolds. So what we really want to show is that on the $n$-dimensional Stein manifold $X:=\mathbb{C}^{n}$ with $\mathcal{F}$ a coherent sheaf and $K$ holomorphically convex compact,

$$
\operatorname{codh}\left(\mathcal{F}_{x}\right) \geq r+1 \text { for all } x \in K \Leftrightarrow H_{K}^{i}(X, \mathcal{F})=0 \text { for all } i \leq r .
$$

The standard proof relies into two main ingredients. First one shows that the homological codimension bounds imply the vanishing of certain extension sheaves. Then one proves that the cohomology groups $H_{K}^{i}(X, \mathcal{F})$ with their natural topology are the strong duals of the spaces $H^{0}\left(K, \mathcal{E} x t_{\mathcal{O}}^{n-i}(\mathcal{F}, \mathcal{O}) \otimes_{\mathcal{O}} \Omega^{n}\right)$, where $\Omega^{n}$ is the sheaf of holomorphic $(n, 0)$ forms on $X$.

Claim I: $\operatorname{codh}\left(\mathcal{F}_{x}\right) \geq r+1$ for all $x \in K \Leftrightarrow \mathcal{E} x t_{\mathcal{O}}^{n-i}(\mathcal{F}, \mathcal{O})=0$ over $K$ and for all $i \leq r$.

Since $K$ is a holomorphically convex compact in $\mathbb{C}^{n}$ we can find an open Stein neighborhood $U$ of $K$ over which there exists an exact sequence of holomorphic free sheaves of the form

$$
0 \longrightarrow \mathcal{O}^{\nu_{r}} \stackrel{\delta_{r}}{\longrightarrow} \mathcal{O}^{\nu_{r-1}} \longrightarrow \cdots \longrightarrow \mathcal{O}^{\nu_{0}} \stackrel{\delta_{0}}{\longrightarrow} \mathcal{F}_{\left.\right|_{U}} \longrightarrow 0 .
$$

We can always choose this sequence to be of minimal length. Let $\check{\mathcal{F}}:=\operatorname{Hom}_{\mathcal{O}}(\mathcal{F}, \mathcal{O})$ be the dual of $\mathcal{F}$. Since $\operatorname{Hom}_{\mathcal{O}}(\cdot, \mathcal{O})$ is a contravariant functor by applying it to (44) we obtain the following sequence over $U$

$$
0 \longrightarrow \check{\mathcal{F}} \stackrel{\delta_{0}^{t}}{\longrightarrow} \mathcal{O}^{\nu_{0}} \stackrel{\delta_{1}^{t}}{\longrightarrow} \mathcal{O}^{\nu_{1}} \longrightarrow \cdots \stackrel{\delta_{r}^{t}}{\longrightarrow} \mathcal{O}^{\nu_{r}} \longrightarrow 0 .
$$

The above complex is not exact-except at $\check{\mathcal{F}}$ and $\mathcal{O}_{\nu_{0}}$. Let us define $\mathcal{E} x t_{\mathcal{O}}^{i}(\mathcal{F}, \mathcal{O}):=\operatorname{kern}\left(\delta_{i+1}^{t}\right) / \operatorname{Im}\left(\delta_{i}^{t}\right)$ for $i>0$. These sheaves measure the degree to which the above complex fails to be exact. They are coherent sheaves on $K$. Let $\operatorname{codh}_{K}(\mathcal{F}):=\inf _{x \in K} \operatorname{codh}\left(\mathcal{F}_{x}\right)$. By the assumption of the $\operatorname{claim} \operatorname{codh}_{K}(\mathcal{F}) \geq r+1$. Then $n-\operatorname{codh}_{K}(\mathcal{F}) \leq n-r-1<n-r$. Hence for all $i \leq r, \mathcal{E} x t_{\mathcal{O}}^{n-i}(\mathcal{F}, \mathcal{O})=0$ over $K$.

Claim II: $H_{K}^{i}(X, \mathcal{F})$ is the strong dual of $H^{0}\left(K, \mathcal{E} x t_{\mathcal{O}}^{n-i}(\mathcal{F}, \mathcal{O}) \otimes \Omega^{n}\right)$.

The proof of the above claim proceeds in several steps. First you consider the case of locally free sheaves $\mathcal{F}$ and you show that i) $H_{K}^{i}(X, \mathcal{F})=0$ if $i \neq n$, ii) $H^{0}(K, \breve{\mathcal{F}})$ with the natural inductive limit topology is a (DFS) space (strong dual of a Fréchet space ) and iii) $H_{K}^{n}(X, \mathcal{F})$ with its natural topology is the strong dual of $H^{0}\left(K, \check{\mathcal{F}} \otimes \Omega^{n}\right)$ (see theorem 5.9 in [27]). Serre's duality theorem plays a critical role in the proofs of the above statements. For a general coherent sheaf $\mathcal{F}$ one uses a short exact sequence of the form $0 \rightarrow \mathcal{G} \rightarrow \mathcal{O}^{s_{0}} \rightarrow \mathcal{F} \rightarrow 0$ over a Stein neighborhood of $K$ (such a sequence always exist since $\mathcal{F}$ is coherent) and apply induction on the homological codimension of $\mathcal{F}$ over $K$ to prove that $H^{0}\left(K, \mathcal{E} x t_{\mathcal{O}}^{n-i}(\mathcal{F}, \mathcal{O}) \otimes \Omega^{n}\right)$ with its natural inductive limit topology is a (DFS) space and then show that $H_{K}^{i}(X, \mathcal{F})$ with its natural topology is the strong dual of $H^{0}\left(K, \mathcal{E} x t_{\mathcal{O}}^{n-i}(\mathcal{F}, \mathcal{O}) \otimes \Omega^{n}\right)$ (see theorem 5.12 in [27]).

Historical remarks: (i)-(iii) in the previous paragraph are special cases of more general theorems proven by Martineau in [20. The passage from locally free sheaves to coherent sheaves is due to Harvey [16].

Theorem 2.1 follows immediately from Claims I and II. 
2.4. Proof of Theorems 1.3, 1.4. Reduction steps. $\alpha$ ) Let us suppose that the first half of Theorem 1.3 is true for $U=X$. This implies that $H_{K}^{0}(X, \mathcal{O})=H_{K}^{1}(X, \mathcal{O})=\{0\}$. Now, let $U$ be any open neighborhood of $K$. Consider the long exact sequence

$$
0 \rightarrow H_{K}^{0}(U, \mathcal{O}) \rightarrow H^{0}(U, \mathcal{O}) \rightarrow H^{0}(U \backslash K, \mathcal{O}) \rightarrow H_{K}^{1}(U, \mathcal{O}) \rightarrow \ldots
$$

From the excision property we know that $H_{K}^{i}(X, \mathcal{O}) \cong H_{K}^{i}(U, \mathcal{O})$ for any open neighborhood $U$ of $K$. Since the former cohomology groups vanish these will imply that $H_{K}^{i}(U, \mathcal{O})=\{0\}$ for $i=0,1$. Hence, $H^{0}(U, \mathcal{O}) \cong H^{0}(U \backslash K, \mathcal{O})$.

$\beta$ ) We need to show now that the theorem is true for $U=X$. If $K$ is a holomorphically convex compact in $X$ such that $\operatorname{codh}\left(\mathcal{O}_{x}\right) \geq 2$ for all $x \in K$, then due to Theorem 2.1 we have that $\Gamma(X \backslash K, \mathcal{O}) \cong \Gamma(X, \mathcal{O})$ or equivalently that $H_{K}^{i}(X, \mathcal{O})=\{0\}$ for $i=0,1$.

Now, let $K$ be as in Theorem 1.2. Apply Theorem 2.1 to its holomorphically convex hull $\hat{K}_{X}$ in $X$. This will imply that $H_{\hat{K}_{X}}^{i}(X, \mathcal{O})=0$ for $i=0,1$. Using these vanishing results and (3) we deduce that $H_{K}^{0}(X, \mathcal{O})=\{0\}$ and $H_{K}^{1}(X, \mathcal{O}) \cong H_{\hat{K}_{X} \backslash K}^{0}(X \backslash K, \mathcal{O})$. The assumption that $X \backslash K$ has no relatively compact branches in $X$ implies that $H_{\hat{K}_{X} \backslash K}^{0}(X \backslash K, \mathcal{O})=\{0\}$ and hence $H_{K}^{1}(X, \mathcal{O})=\{0\}$.

The prove the converse conclusion we refer to Theorem 2.1 and (2).

The proof of Theorem 1.4 will proceed along the same lines.

\section{Equivalence of Theorems 1.4 and 1.5 for Stein spaces}

3.1. Preliminaries on topological properties of complements of compact subsets on connected normal Stein spaces. Let $(X, \mathcal{O})$ be a connected normal (thus irreducible) Stein space of dimension $n \geq 2$. Let $K$ be a compact subset of $X$ and let $\hat{K}$ denote the holomorphically convex hull of $K$ in $X$. Let $\left\{V_{i}\right\}_{i \in I}$ (resp. $\left\{U_{j}\right\}_{j \in J}$ ) be the connected components of $X \backslash K$ that are relatively compact (resp. unbounded) in $X$. Then $X \backslash K=\left(\cup_{i \in I} V_{i}\right) \cup\left(\cup_{j \in J} U_{j}\right)$.

Claim: Let $K$ be a holomorphically convex compact subset of a connected normal Stein space $X$ of dimension $n \geq 2$. Then, $X \backslash K$ has no relatively compact connected components.

Proof of Claim: Suppose on the contrary that $X \backslash K$ has a relatively compact connected component $V$. Now, $X \backslash K$ is open thus normal and $V$ is open, connected with $\bar{V}$ compact. Any $f \in \Gamma(X, \mathcal{O})$ is continuous on $\bar{V}$, hence it attains its maximum on $p \in \bar{V}$. If $p \in V$ then we would be able to find a small, Stein neighborhood $W$ of $p$ such that $f$ attains a local maximum there. Hence $f$ would be constant near $p$ (by the local maximum principle, page 109 in [11]). Since $X$ is irreducible by the Identitätsatz (page 170 in 11]) $f$ should be constant on $X$. Hence $f$ attains its maximum on $\partial V$. But $\partial V \subset K$ and for all $z \in V$ and all $f \in \Gamma(X, \mathcal{O})$ we have: $|f(z)| \leq|f(p)| \leq \sup _{K}|f|$. This last inequality implies that $V \subset \hat{K}=K$ since $K$ is holomorphically convex compact and hence $V=\emptyset$. So $X \backslash K$ has no relatively compact connected components.

Remark: When $X$ is a connected Stein manifold of dimension $n \geq 2$, the above Claim was proved by Laufer (Theorem 9 in [19]).

3.2. Theorem 1.5 for Stein spaces imply Theorem 1.4 (or Corollary 4.4 in $[5]$ ). Let $(X, \mathcal{O})$ be a reduced Stein space and $K$ be a compact set such that $X \backslash K$ has no relatively compact branches in $X$ and furthermore $\operatorname{codh}\left(\mathcal{O}_{X}\right) \geq 2$. When $X$ is not irreducible, then we have $\operatorname{codh}\left(\mathcal{O}_{x}\right)=\inf _{P}\left\{\operatorname{codh}\left(\mathcal{O}_{x}\right)_{P}\right\}$, where $m$ is the maximal ideal in $\mathcal{O}_{x}, \mathcal{P}$ is a prime ideal in $\mathcal{O}_{x}$ and the infimum is taken over all prime ideals $\mathcal{P}$ that contain $m$ (see for example Corollary 3.6, page 42 in [13]). Let us remark here that the condition $\operatorname{codh}\left(\mathcal{O}_{x}\right) \geq 2$ for all $x \in K$ (that appears in Corollary 4.4 in [5]) would guarantee that 1-dimensional irreducible components of $X$ do not intersect $K$.

We shall give an alternative proof of Theorem 1.4 (resp. Corollary 4.4 in [5]) assuming that the version of Theorem 1.5 for Stein spaces is true. Let $X=\cup X_{i}$ be the decomposition of $X$ into irreducible components 
and let $(\hat{X}, \xi)$ be a normalization of $X$. The map $\xi: \hat{X} \rightarrow X$ is a one-sheeted analytic covering map such that $\hat{\mathcal{O}}_{X}=\xi_{*}\left(\mathcal{O}_{\hat{X}}\right)$. Here $\hat{\mathcal{O}}_{X}$ is the sheaf of weakly holomorphic functions on $X$. According to Grauert and Remmert (Global Decomposition Theorem, page 172 in [11), the irreducible components of $X$ are the $\xi$-images of the connected components of $\hat{X}$ and every irreducible component of $X$ contains smooth points of $X$. We write $\hat{X}=\bigsqcup \hat{X}_{i}$, i.e. $\hat{X}$ is the disjoint union of its connected components, which in turn are the normalizations of the irreducible branches of $X$. Since $X \backslash K$ has no relatively compact branches in $X$, $\xi^{-1}(X \backslash K)=\bigsqcup\left(\hat{X}_{i} \backslash \hat{X}_{i} \cap \xi^{-1}(K)\right)$ will have no relatively compact connected components in $\hat{X}$. Hence $\hat{X}_{i} \backslash\left(\hat{X}_{i} \cap \xi^{-1}(K)\right)$ has no relatively compact connected components in $\hat{X}_{i}$, for every $i$. If we could show that $\hat{X}_{i} \backslash\left(\hat{X}_{i} \cap \xi^{-1}(K)\right)$ is connected then we could use the version of Theorem 1.5 and finish the proof of Theorem 1.4.

Some remarks. Let $(X, \mathcal{O})$ be any complex space and $L$ be any compact subset of $X$. Assume that $X=L \cup\left(\cup_{i} V_{i}\right) \cup\left(\cup_{a} U_{a}\right)$ where $V_{i}$ are the relatively compact connected components of $X \backslash L$ and $U_{a}$ the unbounded connected components of $X \backslash L$. Any compact $L^{\prime}$ that contains $L$ satisfies $\left(X \backslash L^{\prime}\right) \cap U_{a} \neq \emptyset$ for each $a$. Take as $L^{\prime}$ the holomorphic convex-hull of $L$ in $X$. If we could show that $X \backslash L^{\prime}$ is connected, then it would easily follow that $X \backslash L \backslash\left(\cup_{i} V_{i}\right)$ is connected.

We want to show that $\hat{X}_{i} \backslash\left(\hat{X}_{i} \cap \xi^{-1}(K)\right)$ is connected; each $\hat{X}_{i}$ is a connected, Stein, normal space and those $\hat{X}_{i}$ that intersect $\xi^{-1}(K)$ are at least 2-dimensional. Let us consider the compact subset $L:=\hat{X}_{i} \cap \xi^{-1}(K)$ of $\hat{X}_{i}$. Let $L^{\prime}$ be the holomorphic convex-hull of $L$ in $\hat{X}_{i}$. Based on the above remarks, to show that $\hat{X}_{i} \backslash L$ is connected it would be sufficient to show that $\hat{X}_{i} \backslash L^{\prime}$ is connected. If the latter set had more than one unbounded connected components, then one could define an $f \in \Gamma\left(\hat{X}_{i} \backslash L^{\prime}, \mathcal{O}\right)$ such that $f=1$ on one of the components and 0 elsewhere on $\hat{X}_{i} \backslash L^{\prime}$. Now, $L^{\prime}$ is holomorphically convex in $\hat{X}_{i}, \operatorname{codh}\left(\mathcal{O}_{y}\right) \geq 2$ for all $y \in L^{\prime}$ and $\hat{X}_{i}$ is Stein. By Theorem 2.1 we have that

$$
\Gamma\left(\hat{X}_{i}, \mathcal{O}\right) \cong \Gamma\left(\hat{X}_{i} \backslash L^{\prime}, \mathcal{O}\right)
$$

By (6) we conclude that $f$ extends to a global section $\hat{f} \in \Gamma\left(\hat{X}_{i}, \mathcal{O}\right)$. Since $\hat{X}_{i}$ is connected, Identitätssatz would imply that $\hat{f} \equiv 0$ on $\hat{X}_{i}$ which is impossible. Hence we can conclude that $\hat{X}_{i} \backslash L^{\prime}$ is connected and thus $\hat{X}_{i} \backslash\left(\hat{X}_{i} \cap\left(\xi^{-1}(K)\right)\right.$ is connected for all $i$ such that $\hat{X}_{i} \cap \xi^{-1}(K) \neq \emptyset$.

All the assumptions of Theorem 1.5 are satisfied for $\hat{X}_{i}$ and $\hat{X}_{i} \cap \xi^{-1}(K)$. Given $s \in \Gamma\left(X \backslash K, \mathcal{O}_{X}\right)$; its pull-back $s \circ \xi \in \Gamma\left(\hat{X} \backslash \xi^{-1}(K), \mathcal{O}_{\hat{X}}\right)$ and its restriction on $\hat{X}_{i} \backslash \hat{X}_{i} \cap \xi^{-1}(K)$ is a holomorphic section there. According to Theorem 1.5 there exist $\sigma_{i} \in \Gamma\left(\hat{X}_{i}, \mathcal{O}\right)$ such that $\sigma_{i}=s \circ \xi$ on $\hat{X}_{i} \backslash \hat{X}_{i} \cap \xi^{-1}(K)$. Hence, there exists a $\sigma \in \Gamma\left(\hat{X}, \mathcal{O}_{\hat{X}}\right)$ such that $\sigma=\sigma_{i}$ on each $\hat{X}_{i}$. Let $\tilde{s}=\xi_{*} \sigma$. Clearly, $\tilde{s}$ is a weakly holomorphic function on $X$ and it is equal to $s$ on $X \backslash K$. Let $A:=\left\{x \in X ; \quad \tilde{s}_{x} \notin \mathcal{O}_{x}\right\}=\operatorname{Supp}\left(\left(\mathcal{O}_{X}+\tilde{s} \mathcal{O}_{X}\right) / \mathcal{O}_{X}\right)$. As $\mathcal{O}_{X}+\tilde{s} \mathcal{O}_{X}$ is a coherent $\mathcal{O}_{X}$-submodule of $\tilde{O}_{X}, A$ is an analytic set in $X$ which is contained in $K$. Hence $A$ is a finite subset of $K$. If $A \neq \emptyset$ then $\operatorname{codh}\left(\mathcal{O}_{y}\right)<2$ for $y \in A$, otherwise $\tilde{s}$ could extend holomorphically in a neighborhood $U$ of $y$, by Corollary 3.3, page 38 in [5]. But this contradicts the assumption that $\operatorname{codh}\left(\mathcal{O}_{x}\right) \geq 2$ for all $x \in K$. Hence $A=\emptyset$, thus $\tilde{s} \in \Gamma(X, \mathcal{O})$. This proves the surjectivity of the restriction mapping $\Gamma(X, \mathcal{O}) \rightarrow \Gamma(X \backslash K, \mathcal{O})$. To prove the injectivity we consider $s \in \Gamma(X, \mathcal{O})$ such that $s=0$ on $X \backslash K$; hence $s=0$ on each $X_{i} \backslash\left(K \cap X_{i}\right)$. By the Identitätssatz we have that $s=0$ on each $X_{i}$, hence $s=0$ on $X$.

Proof of Corollary 1.6. To prove Corollary 1.6 we shall use the above approach, passing to the normalization and assuming Theorem 1.5 for normal Stein spaces. Under the assumptions of Corollary 1.6, we can show that the restriction homomorphism $\Gamma(U, \mathcal{O}) \rightarrow \Gamma(U \backslash K, \mathcal{O})$ is actually an isomorphism. This will imply $H_{K}^{i}(U, \mathcal{O})=0$ for $i=0,1$. By excision we have $H_{K}^{i}(D, \mathcal{O})=0$ for $i=0$, 1 . Hence, the restriction homomorphism $\Gamma(D, \mathcal{O}) \rightarrow \Gamma(D \backslash K, \mathcal{O})$ is an isomorphism.

3.3. Theorem 1.4 implies Theorem 1.5 for Stein spaces. We assume Theorem 1.4 is true. We will show how to derive Theorem 1.5 from it in the case of Stein spaces. Let $\Omega$ be any domain in $X$ and $K \subset \Omega$ be a compact such that $\Omega \backslash K$ is connected. 
Claim: Under the assumptions of Theorem 1.5 on $X, \Omega, K$ we have that $X \backslash K$ has no relatively compact connected components and is connected.

Proof of the Claim: Let $\hat{K}$ be the holomorphically convex hull of $K$ in $X$. We have seen that $X \backslash \hat{K}$ has no relatively compact connected components and that $X \backslash \hat{K}$ is connected (by the results in sections 3.1 and 3.2) consisting only of one unbounded component. Let $\left\{V_{i}\right\}_{i \in I}$ (resp. $\left\{U_{a}\right\}_{a \in J}$ ) be the connected components of $X \backslash K$ that are relatively compact (resp. unbounded) in $X$. Since $X \backslash \hat{K}$ is connected this will imply that the set $J$ consists of a single element. Since $\Omega$ is a neighborhood of $K$ it would intersect every component of $X \backslash K$. We can write $\Omega \backslash K=\Omega \cap(X \backslash K)=\Omega \cap\left(\cup_{i \in I} V_{i} \cup U\right)=\left(\cup_{i \in I} \Omega \cap V_{i}\right) \cup(\Omega \cap U)$. If $I \neq \emptyset$ then we arrive at a contradiction since $\Omega \backslash K$ is connected. Hence $X \backslash K$ has no relatively compact connected components and it is connected.

On a normal space $X$ of dimension $n \geq 2$ we have that $\operatorname{codh}\left(\mathcal{O}_{X}\right) \geq 2$. Hence all the assumptions of Theorem 1.4 are satisfied. Let $s \in \Gamma\left(X \backslash K, \mathcal{O}_{X}\right)$. By theorem 1.4 there exists a unique $\hat{s} \in \Gamma\left(X, \mathcal{O}_{X}\right)$ such that $\hat{s}=s$ on $X \backslash K$. This implies that $H_{K}^{i}(X, \mathcal{O})=0$ for $i=0,1$ and by the excision property that $H_{K}^{i}(\Omega, \mathcal{O})=0$ for $i=0,1$. The latter though imply that $H^{0}(\Omega, \mathcal{O}) \cong H^{0}(\Omega \backslash K, \mathcal{O})$.

3.4. Necessity of conditions in theorems 1.3 or 1.4. Let up point out that the homological codimension (depth) bound in Theorem 1.3 (or Theorem 1.4) is necessary for a Hartogs extension theorem to hold. In [16, Harvey gave an example of an irreducible, Stein surface $X$ in $\mathbb{C}^{4}$ with a single isolated singularity at the origin such that when $K=\{0\}$, there exists a holomorphic function on $X \backslash K$ that can not be extended holomorphically to $X$. Harvey's point was to show that simply requiring the singular Stein space to be of dimension bigger or equal to 2 does not suffice for an extension theorem to hold. Since this example has been the source for interesting phenomena (similar examples had been constructed earlier to show that irreducible Stein varieties whose singular locus has codimension at least 2 are not necessarily normal ([12], Volume II, page 196), we will recall it and also provide a calculation of the homological codimension of the structure sheaf of the variety at 0 in absence of a reference for it.

Let $f: \mathbb{C}^{2} \rightarrow \mathbb{C}^{4}$ be the map described by $f(x, y)=\left(x^{2}, x^{3}, y, x y\right)$. Clearly $f^{-1}(0,0,0,0)=(0,0), f$ is proper and the Jacobian of $f$ has rank 1 at 0 and 2 everywhere else. If we set $X:=f\left(\mathbb{C}^{2}\right)$ then $X$ is a Stein surface in $\mathbb{C}^{4}$ with an isolated singularity at 0 and since $X \backslash\{0\}=f\left(\mathbb{C}^{2} \backslash(0,0)\right)$ is connected $X$ is irreducible. Let us consider the following function on $X \backslash\{0\} ; g\left(z_{1}, z_{2}, z_{3}, z_{4}\right)=\frac{z_{2}}{z_{1}}=x$, if $z_{1} \neq 0$ and $g\left(z_{1}, z_{2}, z_{3}, z_{4}\right)=\frac{z_{4}}{z_{3}}=x$, if $z_{3} \neq 0$. Clearly $g$ is a holomorphic function on $X \backslash\{0\}$. If $g$ could be extended holomorphically across 0 to some function $\tilde{g}$, then one could define a holomorphic map $F: X \rightarrow \mathbb{C}^{2}$ such that $F\left(z_{1}, z_{2}, z_{3}, z_{4}\right)=\left(\tilde{g}\left(z_{1}, z_{2}, z_{3}, z_{4}\right), z_{3}\right)$ that is the inverse of $f$, i.e. $f$ would be a biholomorphism. Hence $g$ can not be extended holomorphically across 0 and therefore $\operatorname{codh}\left(\mathcal{O}_{x, 0}\right) \leq 1$. We shall show that $\operatorname{codh}\left(\mathcal{O}_{X, 0}\right)=1$ by showing that $\operatorname{codh}\left(\mathcal{O}_{X, 0}\right)>0$. We know that $\operatorname{codh}\left(\mathcal{O}_{X, 0}\right)=0$ if and only if every element in $m_{X, 0}$ (the maximal ideal in $\mathcal{O}_{X, 0}$ ) is a zero-divisor in $\mathcal{O}_{X, 0}$. Taking into account that $\mathcal{O}_{X, 0} \cong \mathbb{C}\left\{x^{2}, x^{3}, y, x y\right\}$ and that $m_{X, 0}$ is generated by elements in the maximal ideal of $\mathcal{O}_{\mathbb{C}^{4}}$ at 0 , we immediately see that $\operatorname{codh}\left(\mathcal{O}_{X, 0}\right)$ can not be zero.

Let $\nu$ be the minimal depth (homological codimension) of the rings $\mathcal{O}_{X, x}, x \in \operatorname{Sing} X$. When $\nu=1$ Andersson and Samuelsson gave a necessary and sufficient condition for extendability. More precisely they proved the following:

Theorem 3.1. (Theorem 1.4 ii) in [1]) Assume that $X$ is a Stein space of pure dimension $d$ with globally irreducible components $X^{\ell}$ and let $K$ be a compact subset such that Reg $X^{\ell} \backslash K$ is connected for each $\ell$. Assume $\nu=1$ and let $\chi$ be a cut-off function that is identically equal to 1 in a neighborhood of $K$. There exists a smooth $(d, d-1)$ form a on Reg $X$ such that the function $\phi \in \mathcal{O}(X \backslash K)$ has a holomorphic extension $\Phi$ across $K$ if and only if

$$
\int_{X} \bar{\partial} \chi \wedge a \phi h=0
$$

for all $h \in \mathcal{O}(X)$, and where the integrals exist as principal values at Sing $X$. 
In the case of curves in $\mathbb{C}^{2}$ similar moment conditions were given by Hatziafratis (Theorem 2 in [17]).

Remark: Using Harvey's example we can show why a stronger condition on the homological codimension of $\mathcal{O}_{X}$ is needed in the statement of Corollary 4.4 in [5. There, it is only assumed that $\operatorname{codh}\left(\mathcal{O}_{x}\right) \geq 2$ for all $x \in K$. In the proof provided though, one has to use the fact that $\operatorname{codh}\left(\mathcal{O}_{x}\right) \geq 2$ for all $x \in$ $\hat{K}_{X}$, the holomorphic convex hull of $K$ in $X$. We shall show that we can find a compact subset $K$ in Harvey's surface $X$ such that $X \backslash K$ is connected and $0 \in \hat{K}_{X}$ (recall that $\operatorname{codh}\left(\mathcal{O}_{0}\right)=1$ !). Let $P(r)$ denote the bi-disc of radius $r$. We can construct a compact $L$ in $\mathbb{C}^{2}$ such that: $L:=\overline{P(1)} \backslash T_{\epsilon}$, where $T_{\epsilon}:=\left\{(z, w) ; \quad(\operatorname{Im} z)^{2}+|w|^{2}<\epsilon^{2}\right\}$ for $0<\epsilon<1$ is an open tube about $\mathbb{R} \times\{0\}$. Clearly $0 \notin L$ and $\mathbb{C}^{2} \backslash L$ is connected. In what follows by $\Delta$ we will denote the unit disk in $\mathbb{C}$ with respect to the $z$ or $w$ variable.

Claim: If $g$ is a holomorphic function near $L$ then $g_{\uparrow L}$ has a bounded holomorphic extension $\tilde{g}$ to $P(1)$.

Proof of Claim. Let us set $\tilde{g}(z, w):=\frac{1}{2 \pi i} \int_{|\zeta|=1} \frac{g(z, \zeta)}{\zeta-w} d \zeta$. Clearly $\tilde{g}$ is defined in $P(1)$. When $|z|<1$ and $|\operatorname{Im} z|>\epsilon$, we have $\{z\} \times \bar{\Delta} \subset L$, so $\tilde{g}(z, w)=g(z, w)$ when $|w|<1$ by Cauchy's formula. Since $L$ is connected and $U:=(\{|z|<1,|\operatorname{Im} z|>\epsilon\}) \times\{|w|<1\} \neq \emptyset$ subset of $L$, by the identity principle of holomorphic functions we conclude that $\tilde{g}=g$ on $L$ and hence $\tilde{g}$ is a bounded holomorphic extension of $g$ in $P(1)$.

Let $f: \mathbb{C}^{2} \rightarrow X$ be the parametrization of Harvey's example and let $K:=f(L)$. If $g$ is a holomorphic function on a neighborhood $D$ of $K$ in $X$, it follows that $g$ has a bounded analytic continuation on $f(P(1)) \backslash\{0\}$ since $f$ is a biholomorphism from $\mathbb{C}^{2} \backslash\{(0,0)\}$ to $X \backslash\{0\}$. Hence, $D$ can not be a Stein domain in $X \backslash\{0\}$ and $K$ can not be contained in a holomorphically convex compact in $X \backslash\{0\}$.

3.5. Relatively compact branches. Take $X=: \mathbb{C}^{2}$ and $K=:\left\{\left.(z, w)|1 \leq| z\right|^{2}+|w|^{2} \leq 2\right\}$. Then $\operatorname{codh}\left(\mathcal{O}_{X}\right)=2=\operatorname{dim} X, X$ is normal connected (thus irreducible) and $X \backslash K=U \cup W$ where $U$ is the unit ball and $W=\left\{\left.(z, w)|| z\right|^{2}+|w|^{2}>2\right\}$. Consider a function $f$ such that $f=1$ on $U$ and $f=0$ on $W$. Clearly $f \in H^{0}(X \backslash K, \mathcal{O})$ but $f$ can not be extended to a holomorphic function on $\mathbb{C}^{2}$. One can produce more refined counterexamples where $X \backslash K$ is connected; consider for example as $X:=X_{1} \cup X_{2}$ where $X_{1}$ is the plane in $\mathbb{C}^{3}$ given by $z_{1}=0$ and $X_{2}$ the plane in $\mathbb{C}^{3}$ described by $z_{3}=0$ and let $K:=\left\{\left(z_{1}, z_{2}, z_{3}\right) ; 1 \leq\left|z_{1}\right|^{2}+\left|z_{2}\right|^{2} \leq 2, z_{3}=0\right\}$. Clearly codh $\left(\mathcal{O}_{X}\right)=2$ and $X \backslash K$ is connected. On the other hand $X \backslash K$ has a relatively compact component $U$. Let us define a function $f$ such that $f=z_{1}$ on $U$ and $f=0$ elsewhere on $X \backslash K ; f \in \Gamma(X \backslash K, \mathcal{O})$. Such an $f$ can not be extended holomorphically on $X$. In [1] an example is given (Example 3, Section 8) to show that when $X \backslash K$ has an irreducible component that is relatively compact in $X$ and $\nu=1$ the moment condition (7) is not sufficient to guarantee extendability.

\section{A new proof of a Hartogs extension theorem based on Andreotti-Grauert's Work}

In [2] the following theorem was proved:

Theorem 4.1. (Théorème 15) Let $X$ be a complex space for which there exists a function $\phi>0$, strongly $q$-convex such that the subsets $X_{\epsilon, c}:=\{x \in X \mid \epsilon<\phi(x)<c\}$ are relatively compact for $\epsilon, c>0$. Let $B_{c}:=\{x \in X \mid \phi(x)<c\}$ pour $c>0$. Let $\mathcal{F}$ be a coherent sheaf on $X$. Then the homomorphism

$$
H^{r}(X, \mathcal{F}) \rightarrow H^{r}\left(X \backslash B_{c}, \mathcal{F}\right)
$$

is bijective for $r<\operatorname{codh}(\mathcal{F})-q$ and injective for $r=\operatorname{codh}(\mathcal{F})-q$.

The key ingredient in the proof is the vanishing of the cohomology groups with compact support, $H_{c}^{r}\left(B_{c}, \mathcal{F}\right)$ for all $r \leq \operatorname{codh}(\mathcal{F})-q$. This latter vanishing is obtained via Grauert's bumbing method and some local vanishing of the sheaf cohomology groups with compact support near the boundary of $B_{c}$. When $X$ is a Stein space (or equivalently a 1-complete space) such that $\operatorname{codh}\left(\mathcal{O}_{X}\right) \geq 2$ then from the above theorem we deduce that the restriction homomorphism

$$
H^{0}(X, \mathcal{O}) \rightarrow H^{0}\left(X \backslash B_{c}, \mathcal{O}\right)
$$

is a bijection. From this we can deduce the following theorems 
Theorem 4.2. Let $X$ be a Stein space such that $\operatorname{codh}\left(\mathcal{O}_{X}\right) \geq 2$ and $K$ be a compact subset of $X$. If $X \backslash K$ has no irreducible components that are relatively compact in $X$ then the restriction homomorphism $H^{0}(X, \mathcal{O}) \rightarrow H^{0}(X \backslash K, \mathcal{O})$ is an isomorphism.

Proof. Since $X$ is Stein, there exists a strictly plurisubharmonic function $\phi$ that satisfies the assumptions of Theorem 15 in 2] and for $K$ compact subset of $X$ there exists a $c>0$ such that $K \subset B_{c}$. Let $f \in H^{0}(X \backslash K, \mathcal{O})$. Then the restriction of $f$ on $X \backslash B_{c}$ is a holomorphic section there, and by Theorem 4.1 there exists a unique section $\hat{f} \in H^{0}(X, \mathcal{O})$ such that $\hat{f}=f$ near $X \backslash B_{c}$. Now by assumption each irreducible component $U$ of $X \backslash K$ is unbounded, hence it will intersect $X \backslash \bar{B}_{c}$ i.e. $U \cap X \backslash \bar{B}_{c} \neq \emptyset$. We have $\hat{f}=f$ on the non-empty open subset $U \cap\left(X \backslash \bar{B}_{c}\right)$ of $U$. By the identity principle for holomorphic functions we obtain $\hat{f}=f$ on $U$ and hence $\hat{f}=f$ on $X \backslash K$. As a consequence we obtain that $H_{K}^{i}(X, \mathcal{O})=0$ for $i=0,1$.

Theorem 4.3. Let $X, K$ be as above and let $D$ be a neighborhood of $K$ in $X$. Then the restriction homomorphism $H^{0}(D, \mathcal{O}) \rightarrow H^{0}(D \backslash K, \mathcal{O})$ is an isomorphism.

Proof. As a consequence of the previous theorem we obtained that $H_{K}^{i}(X, \mathcal{O})=0$ for $i=0,1$. By excision we also have that $H_{K}^{i}(D, \mathcal{O})=0$ for $i=0,1$. The vanishing of $H_{K}^{1}(D, \mathcal{O})$ implies the surjectivity of $H^{0}(D, \mathcal{O}) \rightarrow H^{0}(D \backslash K, \mathcal{O})$. The injectivity of the restriction homomorphism follows from the exactness of $0 \rightarrow H_{K}^{0}(D, \mathcal{O}) \rightarrow H^{0}(D, \mathcal{O}) \rightarrow H^{0}(D \backslash K, \mathcal{O}) \rightarrow H_{K}^{1}(D, \mathcal{O})=0$ and the fact that $H_{K}^{0}(D, \mathcal{O})=0$.

Remark: As we have seen in section 3, Theorem 4.3 implies Theorem 1.5 for Stein spaces which in its turn implies Corollary 4.4 in [5].

Using these theorems we can easily prove connectedness of the complements of some compact sets in appropriate Stein spaces. More precisely we obtain the following:

Corollary 4.4. Let $X$ be an irreducible Stein space with $\operatorname{codh}\left(\mathcal{O}_{X}\right) \geq 2$. Let $K$ be a compact subset of $X$ such that $X \backslash K$ has no irreducible components that are relatively compact in $X$. Then $X \backslash K$ is connected.

Proof. Suppose on the contrary that $X \backslash K=U \cup W$ where $U, W$ disjoint open sets. Let $f=1$ on $U$ and $f=0$ on $W$. Clearly $f \in H^{0}(X \backslash K, \mathcal{O})$. By Theorem 4.2 there exists $\hat{f} \in H^{0}(X, \mathcal{O})$ such that $\hat{f}=f$ on $X \backslash K$. By the identity principle we could have that $\hat{f}=1$ on $X$ which is not possible.

Corollary 4.5. Let $X, K$ be as in Theorem 4.3 and let $D$ be a connected neighborhood of $K$. Then $D \backslash K$ is connected.

Proof. Follows along the same lines as the proof of Corollary 4.4 using Theorem 4.3 and the identity principle.

Remark: If $D$ is a neighborhood of $K$, then $D$ intersects every component of $X \backslash K$; hence $D \backslash K$ connected would imply $X \backslash K$ connected.

\section{A $\bar{\partial}$-Approach to Hartogs extension theorems on Stein spaces}

The main goal in this section is to streamline an analytic approach via $\bar{\partial}$ techniques and duality theorems for proving Theorem 1.5 for Stein spaces with arbitrary singularities.

First we need some preliminaries on the existence of smooth cut-off functions on a singular complex space.

5.1. Cut-off functions. A function on a complex space $X$ is $C^{\infty}$ iff it is locally the pull-back of a $C^{\infty}$ function by a local embedding in an open set in some $\mathbb{C}^{N}$. This condition is independent on the choice of the embedding. In the case where there is a proper embedding $\phi: W \rightarrow U^{\text {open }} \subset \mathbb{C}^{N}$ we may choose a cut-off function $\chi^{\prime} \in C_{0}^{\infty}(U)$ with $\chi^{\prime}=1$ on $\overline{\phi(V)}$ for $V \Subset W$ and set $\chi:=\chi^{\prime} \circ \phi$.

When $X$ is a complex space countable at infinity but not necessarily Stein, the proof of the existence of a smooth partition of unity subordinate to an open covering carries over from the manifold case. This implies the existence of smooth cut-off functions in general. 
5.2. The analytic approach; a reduction. Let $(X, \mathcal{O})$ be a connected, normal, Stein space of dimension greater or equal to 2. Let $K$ be a compact subset of $X$ and $D$ an open neighborhood of $K$ in $X$ such that $D \backslash K$ is connected. We need to show that given any $s \in \Gamma(D \backslash K, \mathcal{O})$ there exists unique $\hat{s} \in \Gamma(D, \mathcal{O})$ such that $\hat{s}_{\left.\right|_{D \backslash K}}=s$. We shall need the following Proposition:

Proposition 5.1. Let $X$ be a connected, non-compact normal complex space and $K$ a compact subset of $X$. Let us assume that $K$ has an open neighborhood $\Omega$ in $X$ with the following property $(P)$

For every "nice" $\bar{\partial}$-closed $(0,1)$-form $f$ on $\operatorname{Reg} \Omega$ with $\operatorname{supp}_{X} f:=$ closure $_{X}($ supp $f)$ compact in $\Omega$, the equation $\bar{\partial} u=f$ has a solution on Reg $\Omega$ with $\operatorname{supp}_{X} u:=$ closure $_{X}$ (suppu) compact in $\Omega$.

Then, for every open neighborhood $D$ of $K$ with $D \backslash K$ connected and every $s \in \Gamma(D \backslash K, \mathcal{O})$ there exists a unique $\tilde{s} \in \Gamma(D, \mathcal{O})$ such that $\tilde{s}=s$ on $D \backslash K$.

"Nice" in the above statement means that the form $f$ can be smoothly extended on $U$ for some local embedding $\phi: W \subset \Omega \rightarrow U^{\text {open }} \subset \mathbb{C}^{N}$.

Proof: We choose $\chi \in C_{o}^{\infty}(D \cap \Omega)$ such that $\chi=1$ near $K$ and we define a $(0,1)$ form $f$ on $\operatorname{Reg} \Omega$ as follows:

$$
f=\left\{\begin{array}{ccc}
s \bar{\partial} \chi & \text { on } & \operatorname{supp}(\bar{\partial} \chi) \cap \operatorname{Reg} \Omega \\
0 & \text { on } & \operatorname{Reg} \Omega \backslash \operatorname{supp}(\bar{\partial} \chi)
\end{array}\right.
$$

Let $u$ be a solution to $\bar{\partial} u=f$ on $\operatorname{Reg} \Omega$ with $\operatorname{supp}_{X} u$ compact in $\Omega$. Let $u^{0}, f^{0}$ denote the trivial extensions by zero outside $X \backslash \Omega$ of $u$ and $f$ respectively. Then $\bar{\partial} u^{0}=f^{0}$ on $\operatorname{Reg} X$ and $\bar{\partial} u^{0}=0$ on $\operatorname{Reg} X \backslash \operatorname{supp} f$. Let $U$ be an unbounded connected component of $X \backslash \operatorname{supp} \chi$. Clearly $u^{0}$ is holomorphic on $\operatorname{Reg} X \backslash \operatorname{supp} \chi$, so $u^{0}$ must be identically equal to 0 on $U$. Hence $\operatorname{supp} u^{0} \subset X \backslash U \cap \Omega$.

Let us define $\tilde{s}$ on $\operatorname{Reg} D$ by setting $\tilde{s}:=u^{0}+(1-\chi) s$. Clearly $\bar{\partial} \tilde{s}=0$ on $\operatorname{Reg} D$ and extends as a holomorphic section (still denoted by $\tilde{s}$ ) on $D$ by normality. Since $X$ is connected, $U$ must have nonempty boundary $b U$ which must intersect the boundary of $\operatorname{supp} \chi$. Hence $U \cap(D \backslash K) \neq \emptyset$ and thus $\tilde{s}=s$ on this open set. Since $D \backslash K$ is connected normal (thus irreducible) we obtain $\tilde{s}=s$ on $D \backslash K$ by the identity principle. Uniqueness follows from the fact that $D$ is connected and the identity principle for holomorphic functions.

Hence it remains to prove that we can always find an open neighborhood $\Omega$ of $K$ that satisfies property (P) of the above proposition. Since $K$ is compact on a Stein space we can always find an open, relatively compact Stein subdomain of $X$ that contains $K$. We shall establish below that Property $(\mathrm{P})$ holds for such domains.

5.3. Solvability with compact support. Let us recall the settings and the main theorem in [7]. Let $X$ be a pure $n$-dimensional reduced Stein space, $A$ a lower dimensional complex analytic subset with empty interior containing Sing $X$, the singular locus of $X$. Let $\Omega$ be an open relatively compact Stein domain in $X$ and $L=\widehat{\bar{\Omega}_{X}}$ be the holomorphic convex hull of $\bar{\Omega}$ in $X$. Since $X$ is Stein and $L=\widehat{L_{X}}, L$ has a neighborhood basis of Oka-Weil domains in $X$. Let $X_{0}$ be an Oka-Weil neighborhood of $L$ in $X, X_{0} \subset \subset X$. Then $X_{0}$ can be realized as a holomorphic subvariety of an open polydisk in some $\mathbb{C}^{s}$. Set $\Omega^{*}=\Omega \backslash A$. Let $d_{A}$ be the distance to $A$, relative to an embedding of $X_{0}$ in $\mathbb{C}^{s}$ and let || and $d V$ denote the induced norm on $\Lambda T^{*} \Omega^{*}$, resp. the volume element (different embeddings of neighborhoods of $\bar{\Omega}$ in $\mathbb{C}^{s}$ give rise to equivalent distance functions and norms). For a measurable $(p, q)$ form $u$ on $\Omega^{*}$ let $\|u\|_{N, \Omega}^{2}:=\int_{\Omega^{*}}|u|^{2} d_{A}^{-N} d V$.

In [7] we proved the following theorem:

Theorem 5.2. Let $X, \Omega$ be as above. For every $N_{0} \geq 0$, there exists $N \geq 0$ such that if $f$ is a $\bar{\partial}-$ closed $(p, q)$-form on $\Omega^{*}, q>0$, with $\|f\|_{N, \Omega}<\infty$, there is $v \in L_{p, q-1}^{2 \text {, loc }}\left(\Omega^{*}\right)$ solving $\bar{\partial} v=f$, with $\|v\|_{N_{0}, \Omega^{\prime}}<\infty$ for every $\Omega^{\prime} \subset \subset \Omega$. For each $\Omega^{\prime} \subset \subset \Omega$, there is a solution of this kind satisfying $\|v\|_{N_{0}, \Omega^{\prime}} \leq C\|f\|_{N, \Omega}$, where $C$ is a positive constant that depends only on $\Omega^{\prime}, N, N_{0}$. 
We shall establish a dual version of Theorem 5.2 taking as $A:=\operatorname{Sing} X$. We would like to prove the following theorem

Theorem 5.3. Let $f$ be a $(p, q)$ form defined on Reg $\Omega$ and $\bar{\partial}$-closed there with $0<q<n$, compactly supported in $\Omega$ and such that $\int_{\operatorname{Reg} \Omega}|f|^{2} d_{A}^{N_{0}} d V<\infty$ for some $N_{0} \geq 0$. Then there exists a solution $u$ to $\bar{\partial} u=f$ on Reg $\Omega$ satisfying $\operatorname{supp}_{X} u \Subset \Omega$ and such that

$$
\int_{\operatorname{Reg} \Omega}|u|^{2} d_{A}^{N} d V \leq C \int_{\operatorname{Reg} \Omega}|f|^{2} d_{A}^{N_{0}} d V
$$

where $N$ is a positive integer that depends on $N_{0}$ and $\Omega$ and $C$ is a positive constant that depends on $N_{0}, N, \Omega$ and supp $f$.

Proof of theorem 5.3. The proof is inspired by the proof of Serre's duality theorem on complex manifolds. We shall apply Theorem 5.2 twice for forms of different bidegree and for $A=\operatorname{Sing} X$. Let $N_{0}$ be as in Theorem 5.3. Then according to Theorem 5.2 for the pair $(X, \Omega)$ and for $N_{0}+2$ there exists $N_{1}(>>$ $\left.N_{0}+2\right)$ such that if $F \in L_{n-p, n-q}^{2, l o c}(\operatorname{Reg} \Omega), \bar{\partial} F=0$ on $\operatorname{Reg} \Omega$ with $\|F\|_{N_{1}, \Omega}<\infty$ then, there exists $a \in L_{n-p, n-q-1}^{2, l o c}(\operatorname{Reg} \Omega)$ satisfying $\bar{\partial} a=F$ on $\operatorname{Reg} \Omega$ and such that $\|a\|_{N_{0}+2, Y}<\infty$ for all $Y \Subset \Omega$. Similarly, for the above $N_{1}$ there exists an $N\left(>>N_{1}\right)$ such that if $G \in L_{n-p, n-q+1}^{2, l o c}(\operatorname{Reg} \Omega)$, $\bar{\partial}$-closed on $\operatorname{Reg} \Omega$ with $\|G\|_{N, \Omega}<\infty$ then, there exists a $b \in L_{n-p, n-q}^{2, l o c}(\operatorname{Reg} \Omega)$ satisfying $\bar{\partial} b=G$ on $\operatorname{Reg} \Omega$ and such that $\|b\|_{N_{1}, Y}<\infty$ for all $Y \Subset \Omega$.

Let $f, N_{0}$ be as in Theorem 5.3 and $N, N_{1}$ be chosen as above. Let us define

$$
\mathcal{H}_{N}:=\left\{w \in L_{n-p, n-q+1}^{2, l o c}(\operatorname{Reg} \Omega):\|w\|_{N, \Omega}<\infty\right\}
$$

and $\mathcal{H}_{N_{1}}^{\text {loc }}:=\left\{v \in L_{n-p, n-q}^{2, l o c}(\operatorname{Reg} \Omega):\|v\|_{N_{1}, Y}<\infty\right.$ for all $\left.Y \Subset \Omega\right\}$. Consider the following map:

defined by

$$
L_{f}: \mathcal{H}_{N} \cap \operatorname{kern}(\bar{\partial}) \rightarrow \mathbb{C}
$$

$$
L_{f}(w):=(-1)^{p+q+1} \int_{\operatorname{Reg} \Omega} v \wedge f
$$

where $v \in \mathcal{H}_{N_{1}}^{\text {loc }}$ is a solution to $\bar{\partial} v=w$ on $\operatorname{Reg} \Omega$ (such a solution always exists by Theorem 5.2).

First of all we need to show that $L_{f}$ is well-defined, i.e. independent of the choice of the solution $v \in \mathcal{H}_{N_{1}}^{\text {loc }}$ to the equation $\bar{\partial} v=w$ on $\operatorname{Reg} \Omega$. It suffices to show that $\int_{\operatorname{Reg} \Omega} v \wedge f=0$ when $v \in \mathcal{H}_{N_{1}}^{\text {loc }}$ and $\bar{\partial} v=0$ on $\operatorname{Reg} \Omega$. Clearly $\bar{\partial} v=0$ on $\operatorname{Reg} \Omega$ and $\|v\|_{N_{1}, V}<\infty$. Hence according to what was discussed above there exists an $a \in L_{n-p, n-q-1}^{2, l o c}(\operatorname{Reg} \Omega)$ satisfying $\bar{\partial} a=v$ on $\operatorname{Reg} \Omega$ and such that $\|a\|_{N_{0}+2, Y}<\infty$ for all $Y \Subset \Omega$. Let $\chi_{\delta} \in C^{\infty}(\operatorname{Reg} \Omega)$ such that $0 \leq \chi_{\delta} \leq 1, \chi_{\delta}(z)=1$ when $d_{A}(z)>\delta, \quad \chi_{\delta}(z)=0$ if $d_{A}(z) \leq \frac{\delta}{2}$ and such that $\left|\bar{\partial} \chi_{\delta}\right| \leq \frac{C}{\delta}$ on $\operatorname{Reg} \Omega$, for some positive constant $C$ independent of $\delta$. Then $L_{f}(z)=\lim _{\delta \rightarrow 0} \int_{\operatorname{Reg} \Omega} \chi_{\delta} v \wedge f=$ $\lim _{\delta \rightarrow 0} \int_{\operatorname{Reg} \Omega} \chi_{\delta} \bar{\partial} a \wedge f=-\lim _{\delta \rightarrow 0} \int_{\operatorname{Reg} \Omega} \bar{\partial} \chi_{\delta} \wedge a \wedge f$. The last equality follows from integration by parts arguments; the form $\chi_{\delta} f$ has compact support on Reg $\Omega$ but is not really smooth, so a smoothing argument is needed in order to apply the standard Stokes' theorem. Let $I_{\delta}:=\int_{\operatorname{Reg} \Omega} \bar{\partial} \chi_{\delta} \wedge a \wedge f$. By Cauchy-Schwarz inequality we have

$$
\left|I_{\delta}\right|^{2} \leq\left(\int_{\text {supp } f \cap \operatorname{supp}\left(\bar{\partial} \chi_{\delta}\right)}\left|d_{A} \bar{\partial} \chi_{\delta}\right|^{2}|f|^{2} d_{A}^{N_{0}} d V\right)\left(\int_{\left\{\chi_{\delta}<1\right\} \cap \operatorname{supp} f}|a|^{2} d_{A}^{-\left(N_{0}+2\right)} d V\right)=A \cdot B
$$

But $B$ is finite and $A \rightarrow 0$ as $\delta \rightarrow 0$ since $\left|d_{A} \bar{\partial} \chi_{\delta}\right| \leq C$ and $\int_{\operatorname{Reg} \Omega}|f|^{2} d_{A}^{N_{0}} d V<\infty$. Hence $\lim _{\delta \rightarrow 0} I_{\delta}=0$ and thus $L_{f}$ is well-defined. 
Clearly $L_{f}$ is a linear map. By Cauchy-Schwarz we have

$$
\left|L_{f}(w)\right| \leq C_{0}\left(\int_{\operatorname{supp} f}|v|^{2} d_{A}^{-N_{1}} d V\right)^{\frac{1}{2}}\left(\int_{\operatorname{Reg} \Omega}|f|^{2} d_{A}^{N_{0}} d V\right)^{\frac{1}{2}}
$$

To obtain this inequality we used the fact that $N_{1}>>N_{0}$ and hence $d_{A}^{N_{1}-N_{0}} \leq C_{0}$ on $\bar{\Omega}$. We want to show that $L_{f}$ factors into a bounded linear functional on a subspace $\mathcal{A}$ of $H_{N} \cap \operatorname{kern}(\bar{\partial})$. Recall that the following lemma was proven in [7] using the open mapping theorem for Fréchet spaces.

Lemma 5.4. (Lemma 4.2 in [7) Let $M$ be a complex manifold and let $E$ and $F$ be Fréchet spaces of differential forms (or currents) of type $(p, q-1),(p, q)$, whose topologies are finer (possibly strictly finer) than the weak topology of currents. Assume that for every $f \in F$, the equation $\bar{\partial} u=f$ has a solution $u \in E$. Then, for every continuous seminorm $p$ on $E$, there is a continuous seminorm $q$ on $F$ such that the equation $\bar{\partial} u=f$ has a solution with $p(u) \leq q(f)$ for every $f \in F, q(f)>0$.

Let $p(v):=\left(\int_{\operatorname{supp} f}|v|^{2} d_{A}^{-N_{1}} d V\right)^{\frac{1}{2}}$. Using the lemma in our situation, given the seminorm $p$ there exists an open set $W \Subset \Omega$ (that depends on the supp $f$ ) such that for all $w \in \mathcal{H}_{N} \cap \operatorname{kern}(\bar{\partial})$ with $q(w)=$ $\left(\int_{W}|w|^{2} d_{A}^{-N} d V\right)^{\frac{1}{2}}>0$ there exists a solution $v$ to $\bar{\partial} v=w$ on $\operatorname{Reg} \Omega$ and a positive constant $C$ satisfying

$$
\left(\int_{\operatorname{supp} f}|v|^{2} d_{A}^{-N_{1}} d V\right)^{\frac{1}{2}} \leq C q(w)
$$

If $q(w)=0$ then the same argument will imply that for every $\epsilon>0$ there exists a solution $v_{\epsilon}$ to $\bar{\partial} v_{\epsilon}=w$ on $\operatorname{Reg} \Omega$ with $p\left(v_{\epsilon}\right)<\epsilon$. Then for such a $w$ we have: $\left|L_{f}(w)\right| \leq C_{0} \epsilon \int_{\operatorname{Reg} \Omega}|f|^{2} d_{A}^{N_{0}} d V$. Here we used the fact that $L_{f}(w)$ is well-defined independent of the choice of the solution $v \in \mathcal{H}_{N_{1}}^{n-q, l o c}(\Omega)$ to $\bar{\partial} v=w$. Taking the limit as $\epsilon \rightarrow 0$ we get that

$$
\left|L_{f}(w)\right|=0 \leq C_{0} q(w)\left(\int_{\operatorname{Reg} \Omega}|f|^{2} d_{A}^{N_{0}} d V\right)^{\frac{1}{2}}
$$

Combining (8), (9), (10) we obtain for all $w \in \mathcal{H}_{N} \cap \operatorname{kern}(\bar{\partial})$

$$
\left|L_{f}(w)\right| \leq C_{0} C q(w)\left(\int_{\operatorname{Reg} \Omega}|f|^{2} d_{A}^{N_{0}} d V\right)^{\frac{1}{2}}
$$

From (11) we see that $L_{f}(w)$ depends only on $w_{\uparrow W}$. Indeed, let $w, w^{\prime} \in \mathcal{H}_{N} \cap \operatorname{kern}(\bar{\partial}) \operatorname{such}$ that $w_{\uparrow W}=w_{\uparrow W}^{\prime}$. Then $L_{f}(w)=L_{f}\left(w-w^{\prime}+w^{\prime}\right)=L_{f}\left(w-w^{\prime}\right)+L_{f}\left(w^{\prime}\right)$. From (11) we obtain that $\left|L_{f}\left(w-w^{\prime}\right)\right| \leq$ $C_{0} C q\left(w-w^{\prime}\right)\left(\int_{\operatorname{Reg} \Omega}|f|^{2} d_{A}^{N_{0}} d V\right)^{\frac{1}{2}}$. But $q\left(w-w^{\prime}\right)=0$ as $w-w^{\prime}=0$ on $W$. Hence $L_{f}\left(w-w^{\prime}\right)=0$ and thus $L_{f}(w)=L_{f}\left(w^{\prime}\right)$. Hence $L_{f}$ factors to a well-defined bounded linear functional on $\mathcal{A}:=\left\{w_{\uparrow W} ; \quad w \in\right.$ $\left.\mathcal{H}_{N} \cap \operatorname{kern}(\bar{\partial})\right\} \subset \mathcal{H}_{N}(W)$. Here $\mathcal{H}_{N}(W):=\left\{w \in L_{n-p, n-q+1}^{2, l o c}(W):\|w\|_{N, W}<\infty\right\}$.

We make a norm-preserving extension of the above functional $L_{f}$ to $\mathcal{H}_{N}(W)$. Let us call $\tilde{L}_{f}$ the extended functional. By Riesz representation theorem there exists a $u^{\prime} \in \mathcal{H}_{N}(W)$ such that for all $w \in \mathcal{H}_{N}(W)$ we have

$$
\tilde{L}_{f}(w)=\int_{\operatorname{Reg} W}<w, u^{\prime}>d_{A}^{-N} d V
$$


Set $u:=d_{A}^{-N} \mp u^{\prime}$ on $\operatorname{Reg} W$ (here $*$ is the Hodge-star operator) and extend by zero outside $\bar{W}$. We claim that $u$ is the desired solution of Theorem 5.3. Certainly $\operatorname{supp} u \Subset \Omega$ and $\int_{\operatorname{Reg} \Omega}|u|^{2} d_{A}^{N} d V=$ $\int_{\operatorname{Reg} W}\left|u^{\prime}\right|^{2} d_{A}^{-N} d V<\infty$, since $u^{\prime} \in \mathcal{H}_{N}(W)$. We can control the weighted $L^{2}$ norm of $u^{\prime}$ in terms of the weighted $L^{2}$-norm of $f$ taking into consideration the following:

$$
\begin{aligned}
& \left(\int_{\operatorname{Reg} W}\left|u^{\prime}\right|^{2} d_{A}^{-N} d V\right)^{\frac{1}{2}}=\left\|\tilde{L}_{f}\right\|=\left\|L_{f \nmid \mathcal{A}}\right\|= \\
= & \sup \left\{\left|L_{f}(w)\right|: w \in \mathcal{A} \text { with } q(w) \leq 1\right\} \leq C_{0} C\left(\int_{\operatorname{Reg} \Omega}|f|^{2} d_{A}^{N_{0}} d V\right)^{\frac{1}{2}} .
\end{aligned}
$$

It remains to show that $\bar{\partial} u=f$ on $\operatorname{Reg} \Omega$. Let $\phi \in C_{0,(n-p, n-q)}^{\infty}(\operatorname{Reg} \Omega)$ be a smooth compactly supported form of bidegree $(n-p, n-q)$ on $\operatorname{Reg} \Omega$. We need to show that

$$
\int_{\operatorname{Reg} \Omega} \bar{\partial} \phi \wedge u=(-1)^{p+q+1} \int_{\operatorname{Reg} \Omega} \phi \wedge f
$$

But $\phi \in \mathcal{H}_{N_{1}}^{\text {loc }}, \bar{\partial} \phi \in \mathcal{H}_{N}$ and $\bar{\partial} \phi_{\Gamma W} \in \mathcal{A}$. Therefore from the definition of $L_{f}$ we have that

$$
\tilde{L}_{f}\left(\bar{\partial} \phi_{\uparrow W}\right)=L_{f}\left(\bar{\partial} \phi_{\uparrow W}\right)=(-1)^{p+q+1} \int_{\operatorname{Reg} \Omega} \phi \wedge f
$$

On the other hand from (12) we have that

$$
\tilde{L}_{f}\left(\bar{\partial} \phi_{\mid W}\right)=\int_{\operatorname{Reg} W} \bar{\partial} \phi \wedge u=\int_{\operatorname{Reg} \Omega} \bar{\partial} \phi \wedge u
$$

Putting the last two equalities together we obtain (14).

The last part of Theorem 5.3 follows directly from the definition of $u$ and (13).

Remark: We can be more precise about the dependence of $\operatorname{supp} u$ and the constant $C$ (that appears in Theorem 5.3) on supp $f$. Let $X, \Omega$ be as in theorem 5.3 and let $N_{0}$ be a non-negative integer. There exists a positive integer $N$ that depends on $N_{0}$ and $\Omega$ such that the following is true: For every compact $K \subset \Omega$, there exists a compact $K^{\prime} \subset \Omega$ and a positive constant $C$ that depends on $K, N, N_{0}, \Omega$ such that for every $(p, q)$ form $f$ with supp $f \subset K$ and $\int_{K \backslash A}|f|^{2} d_{A}^{N_{0}} d V<\infty, \bar{\partial}$-closed on Reg $\Omega$, there exists a solution $u$ to $\bar{\partial} u=f$ on $\operatorname{Reg} \Omega$ with $\operatorname{supp} u \subset K^{\prime}$ satisfying

$$
\int_{K^{\prime} \backslash A}|u|^{2} d_{A}^{N} d V \leq C \int_{K \backslash A}|f|^{2} d_{A}^{N_{0}} d V
$$

The proof follows along the same lines as the proof of Theorem 5.3, by taking as $p(v):=\left(\int_{K \backslash A}|v|^{2} d_{A}^{-N_{1}} d V\right)^{\frac{1}{2}}$. Then there exist an open, relatively compact subset $W$ of $\Omega$ (that depends on $K$ ) a seminorm $q(w):=$ $\int_{W \backslash A}|w|^{2} d_{A}^{-N} d V$ and a positive constant $C^{\prime}$ (that depends on $K$ ) such that the equation $\bar{\partial} v=w$ has a solution $v$ satisfying $p(v) \leq C^{\prime} q(w)$. The rest of the proof of Theorem 5.3 carries over. The compact $K^{\prime}$ is chosen to be $K^{\prime}:=\operatorname{closure}(W)$ and the constant $C=C^{\prime} C_{0}$ where $C_{0}$ depends on $N, N_{0}, \Omega$. 


\section{REFERENCES}

[1] M. Andersson and H. Samuelsson, Koppelman formulsa and the $\bar{\partial}$-equation on an analytic space, preprint available at http://www.arxiv.org arXiv:0801.0710v3.

[2] A. Andreotti and H. Grauert, Théorèmes de finitude pour la cohomologie des espaces complexes, Bull. Soc. Math. France, vol. 90, (1962), 193-259.

[3] A. Andreotti and D. Hill, E. E. Levi convexity and the Hans Lewy problem. Part I: reduction to vanishing theorems, Annali della Scuola Normale Superiore di Pisa, tome 26, no. 2, (1972), 325-363.

[4] A. Andreotti and E. Vesentini, Carlemann estimates for the Laplace-Beltrami equation on complex manifolds, Publ. Math. Inst. Hautes tudes Sci., 25 (1965), 81-130.

[5] C. Bănică and O. Stănăşilă, Algebraic methods in the global theory of complex spaces, John Wiley and Sons, London-New York Sidney, (1976).

[6] M. Coltoiu, A supplement to a theorem of Merker and Porten: a short proof of Hartogs extension theorem for $(n-1)$ complete complex spaces, preprint available at http://arxiv.org arXiv:0811.2352.

[7] J. E. Fornæss, N. Øvrelid and S. Vassiliadou, Semiglobal results for $\bar{\partial}$-on a complex space with arbitrary singularities, Proc. of Amer. Math. Soc., vol. 133, no. 8, (2005), 2377-2386.

[8] H. Grauert, Ein Theorem der analytischen Garbentheorie und die Modulraüme komplexer Structuren, Publ.Math. Inst. Hautes Etud. Sc., no 5, (1960), 5-64.

[9] H. Grauert, T. Peternell and R. Remmert, Several Complex Variables VII, Encyclopaedia of Mathematical Sciences, Volume 74, (1994), Springer-Verlag.

[10] H. Grauert and O. Riemenschneider, Kählersche Mannigfltigkeiten mit hyper-q-konvexen Rand, Problems in analysis (A Symposium in Honor of S. Bochner, Princeton 1969). Princeton University Press, Princeton (1970), 61-79.

[11] H. Grauert and R. Remmert, Coherent Analytic Sheaves, Grundlehren der mathematischen Wissenschaften 265, SpringerVerlag, 1984.

[12] R. Gunning Introduction to holomorphic functions of several variables, I, II, III, Wadsworth and Brooks/Cole Mathematics Series, (1990).

[13] R. Hartshorne, Local cohomology, A seminar given by A. Grothendieck, Harvard, University, 1961, Lecture Notes in Mathematics, 41, Springer-Verlag, (1967).

[14] R. Hartshorne, Algebraic Geometry, Graduate text in Mathematics , 52, Springer-Verlag, 1977.

[15] L. Hörmander, An introduction to Complex Analysis in Several Variables, North-Holland Mathematical Library, 3rd edition, (1990).

[16] R. Harvey, The theory of hyperfunctions on totally real subsets of complex manifolds with applications to extension problems, American Jour. of Math., vol. 91, no. 4, (1969), 853-873.

[17] T. Hatziafratis, Fourier-Laplace transforms and related questions on certain analytic varieties in $\mathbb{C}^{2}$, J. Math. Anal. Appl. 305, (2005), 722-742.

[18] N. Jacobson, Basic Algebra II, 2nd edition, W. H. Freeman and Company, New York, (1989).

[19] H.B. Laufer, Some remarks about a theorem of Hartogs, Proc. of the AMS, vol. 17, no 6, (1966), 1244-1249.

[20] A. Martineau, Les hyperfunctions de M. Sato, Seminaire Bourbaki, vol. 13, (1960-1961), 214-01-214-13.

[21] J. Merker and E. Porten, The Hartogs extension theorem on $(n-1)$-complete complex spaces, available at http://www.arxiv.org arXiv:0704.3216

[22] N. Øvrelid and S. Vassiliadou, Semiglobal results for $\bar{\partial}$ on complex spaces with arbitrary singularities, Part II, in preparation.

[23] H. Rossi, Vector fields on analytic spaces, The Annals of Math., vol. 78 no. 3, 1963, 455-467.

[24] J. Ruppenthal, A $\bar{\partial}$ theoretical proof of Hartogs extension theorem on Stein spaces with isolated singularities, J. Geom. Analysis, 18, no. 4, (2008), 1127-1132.

[25] J. Ruppenthal. $A \bar{\partial}$ theoretical proof of Hartogs extension theorem on $(n-1)$-complete spaces, preprint available at http://arxiv.org arXiv:0811.1963

[26] Y. T. Siu, Analytic sheaf cohomology groups of dimension $n$ of $n$-dimensional complex spaces, Trans. Amer. Math. Soc., 143, (1969), 77-94.

[27] Y. T. Siu and G. Trautmann, Gap sheaves and extension of coherent complex analytix sheaves, Lecture Notes in Mathemetics, Vol. 172, Springer-Verlag 1971.

[28] K. Takegoshi, Relative vanishing theorems in analytic spaces, Duke Math. Journ. vol. 52, no.1, (1985), 273-279.

Dept. of Mathematics, University of Oslo, P.B 1053 Blindern, Oslo, N-0316 NorWAy

Dept. of Mathematics, Georgetown University, Washington, DC 20057 USA

E-mail address: nilsov@math.uio.no, sv46@georgetown.edu 